\title{
EESE: Energy-Efficient Communication between Satellite Swarms and Earth Stations
}

\author{
Yuan An, Jiancheng Li \\ State key Lab of \\ Astronautic Dynamics, \\ Xi' an Satellite Control Center, \\ Xi' an 710043, China \\ 9112533@qq.com
}

\author{
Weiwei Fang \\ School of Computer \\ and Information Technology, \\ Beijing Jiaotong University, \\ Beijing 100044, China \\ fangvv@gmail.com
}

\author{
Baohua Wang, Qiwang Guo, Jing Li, Xiaolong Li, Xing Du \\ State key Lab of \\ Astronautic Dynamics, \\ Xi'an Satellite Control Center, \\ Xi' an 710043, China \\ satelliteswarm@xascc.gov.cn
}

\begin{abstract}
The promising projects of satellite swarm have been studied by scientists from NASA, ESA and other institutes around the world. With a massive number of pico-class, low-power and low-weight space-crafts like pico-satellites, nano-satellites, Swarm can exploring outer space environment or taking other tasks that are hard to be fulfilled by common satellites. The other merit of using swarm than common satellite is cost reduction. Besides all the advantages of satellite swarm, developing swarmbased satellite systems from conceptualization to validation is a complex multi-disciplinary activity. Swarm is commonly energyconstrained, so one of the key challenges is how to achieve energyefficient data transmission between the satellite swarm and terrestrial terminal stations. By employing Lyapunov optimization, we present an online control algorithm called EESE for optimally dispatching traffic load among different satellite-ground links to minimize overall energy consumption over time. Our algorithm is able to independently and simultaneously make control decisions about traffic dispatching on ISLs and UDLs to offer provable energy and delay guarantees, without requiring any statistical information of traffic arrivals and link conditions. Rigorous analysis has demonstrated the performance and robustness of our algorithm.
\end{abstract}

Keywords-Satellite Swarm, Optimal Control, Dynamic Scheduling, Wireless Communication, Energy Efficiency.

\section{INTRODUCTION}

To enable robust space exploration, astronautic researchers are exploiting principles and techniques that help spacecraft systems become more resilient through self-organizing and automatic adaptation. Inspired by the swarming behaviors of animals in nature, they have recently proposed to build swarmbased systems that is comprised of thousands of pico-class, low-power, and low-weight satellite units working together for exploration missions [1]. Example projects include ANTS of NASA [2], APIES of ESA [3] and SeeMe of DARPA [4].

Generally, a swarm is consisted of several sub-swarms, which are temporal groups organized to perform a particular task. Each swarm group has a group leader (i.e.,"ruler"), and a large number of "workers" carrying a specialized instrument. The ruler is responsible for coordinating its workers to cooperate on area monitoring and data gathering. Besides, there are some "messengers" in the swarm to coordinate communications among rulers, works, and earth stations. Although with different roles and responsibilities, all the three types of satellites rely primarily on power from the sun for data gathering, data processing, and data communication. However, the on-board solar panels cannot be very large for the small satellites [5]. Therefore, it is of great necessity to take the energy efficiency issue into consideration when designing such a swarm system.

To address the energy challenges of satellite swarms, we propose a novel online control algorithm, EESE, based on Lyapunov optimization techniques [6] to make traffic dispatching decisions in the context of satellite-ground communications , which offers significant potentials on reducing energy consumption for data transmission over UDLs (Up-Down-Links). Specifically, this algorithm aims to reduce energy consumption by making control decisions on: (1) how to dispatch the traffic load from the workers over ISLs (Inter-Satellite-Links) to the messengers, and (2) how to choose a suitable UDL for a given messenger to transmit the aggregated data from it to ground. Our algorithm does not require any prior knowledge of the system statistics or any prediction on traffic arrivals and link conditions. Moreover, it is computationally efficient and easy to be implemented in practical systems. It can obtain a timeaverage energy consumption within a deviation of $\mathcal{O}(1 / \mathrm{V})$ from optimality, while bounding the traffic service delay by $\mathcal{O}(V)$, where $V$ is a non-negative control parameter representing a design knob of the energy-delay tradeoff, i.e., how much we shall emphasize the energy minimization compared to transmission delays. We thoroughly analyze the performance of EESE with rigorous theoretical analysis.

The rest of this paper is organized as follows: Section II describes the theoretical model, and formulates the objective problem. Section III presents the EESE algorithm, and provides an analysis on the performance bound and robustness of EESE. Finally, Section IV concludes the paper.

\section{Problem Formulation}

We consider a satellite swarm which has $I$ heterogeneous workers denoted by $W=\left\{W_{1}, \ldots, W_{I}\right\}$, as well as $J$ homogeneous messengers denoted by $M=\left\{M_{1}, \ldots, M_{J}\right\}$. The whole swarm system operates in discrete time with unit time slots $t \in\{0,1,2, \ldots\}$. In every time slot $t$, we denote the amount of newly generated data at $W_{i}$ by $A_{i}(t)$, where $\mathbf{A}(t)=\left(A_{1}(t), \ldots, A_{I}(t)\right)$ denotes the arrival vector. We assume that $\mathbf{A}(t)$ are independent identically distributed (i.i.d.) over time slots with $\mathbb{E}\{\mathbf{A}(t)\}=\lambda \triangleq\left(\lambda_{1}, \ldots, \lambda_{I}\right)$. We also assume that there exists a finite maximum $A_{\max }$ such that $0 \leq A_{i}(t) \leq A_{\max }$ for all $t$ and all $i \in\{1, \ldots, I\}$. However, 
we do not assume any priori knowledge of the statics of $A_{i}(t)$. For example, $A_{i}(t)$ could be a Markov-modulated process with time-varying instantaneous rates where the transition probabilities between different state are unknown. This models a scenario with unpredictable and time-varying traffic arrivals.

In a swarm, the worker $W_{i}$ continuously gathers and temporally stores data information obtained by specialized exploring instruments. It then decides how to distribute the queued data to the messengers for transmitting to ground. To model this decision, we use $\mu_{i j}(t)$ to denote the amount of data traffic sent from $W_{i}$ to $M_{j}$ at time $t$, and use $\mu_{i}(t)=\left(\mu_{i 1}(t), \ldots, \mu_{i J}(t)\right)$ to denote the vector of traffic load dispatching rates at $W_{i}$. We assume that in every $t, \mu_{i}(t)$ must be drawn from some general feasible set $\mathcal{R}_{i}$, i.e., $\mu_{i}(t) \in \mathcal{R}_{i}$ for all $t$. We assume that each set $\mathcal{R}_{i}$ contains the constraint that $0 \leq \sum_{j=1}^{J} \mu_{i j}(t) \leq \mu_{\max }$ for some finite constant $\mu_{\max }$. It must be noted here that this assumption is quite general for many typical scenarios.

The current satellite swarm prototypes generally use two different technologies for data communication, i.e., the commercial-off-the-shelf wireless technology (e.g., IEEE 802.11 [7]) for inter-satellite communications within the swar$\mathrm{m}$, and the high-frequency microwave wireless technology (e.g., UHF/C/X/Ku/Ka band [8]) for satellite-ground communications between swarm messengers and earth stations. It is obvious that the latter can bring about energy consumption considerably higher than the former, and has significant impacts on the energy consumption and operational lifetime of messengers. In the same time slot, a messenger may have more than one accessible earth stations at different locations, and the UDLs between them have distinct characteristics, including available duration and error rate [9]. Hence, there exist opportunities for energy saving for the swarm by scheduling data transmission onto those UDLs currently with high quality and low cost. Let $\alpha_{j}(t)$ denotes the decision of messenger $j \in 1, \ldots, J$ on the earth station selection in time slot $t$. Then, the service rates (i.e., throughput) of messenger $j$ for slot $t$ can be given as $X_{j}(t)=X_{j}\left(\alpha_{j}(t)\right)$. We assume a maximum transmission rate $X_{\max }$, regardless of $\alpha(t)$, so that $0 \leq X_{j}(t) \leq X_{\max }$. Besides, the energy consumption for transmitting data for $j$ can be given as $f_{j}(t)=\frac{K * X_{j}(t)}{E_{j}(t)}$, where $K$ is the coefficient of energy consumption for transmitting per unit data, and $E_{j}(t)=E_{j}\left(\alpha_{j}(t)\right)$ is the success rate of selected link in slot $t[10]$.

Let $\mathbf{Q}(t)=\left(W_{i}(t), M_{j}(t), i \in\{1, \ldots, I\}, j \quad \in\right.$ $\{1, \ldots, J\}), t=0,1, \ldots$ be the vector denoting the data traffic queued at the workers and the messengers at time slot $t$, we can capture the following queueing dynamics over time:

$$
\begin{aligned}
& W_{i}(t+1)=\max \left[W_{i}(t)-\sum_{j=1}^{J} \mu_{i j}(t), 0\right]+A_{i}(t) \\
& M_{j}(t+1)=\max \left[M_{j}(t)-X_{j}(t), 0\right]+\sum_{i=1}^{I} \mu_{i j}(t)
\end{aligned}
$$

In the following, we assume that satellites can estimate the unfinished traffic load in their queues accurately, and the case when such estimation has errors will be discussed in Section III. Throughout the paper, we use the following definition of queue stability:

$$
\bar{Q} \triangleq \lim _{T \rightarrow \infty} \frac{1}{T} \sum_{t=0}^{T-1}\left[\mathbb{E}\left\{\sum_{i=1}^{I} W_{i}(t)\right\}+\mathbb{E}\left\{\sum_{j=1}^{J} M_{j}(t)\right\}\right]<\infty
$$

Our objective is to design a flexible and robust online control policy that automatically adapts to the time-varying systems (in terms of traffic arrivals and link conditions) by making decisions on $\mu_{i j}$ and $\alpha_{j}(t)$ for solving the following stochastic minimization problem:

$$
\begin{aligned}
& \min _{\mu_{i j}, \alpha_{j}(t)} \bar{F} \triangleq \lim _{T \rightarrow \infty} \frac{1}{T} \sum_{t=0}^{T-1} \mathbb{E}\left\{\sum_{j=1}^{J} f_{j}(t)\right\} \\
& \text { s.t. } \\
& 0 \leq A_{i}(t) \leq A_{\max }, \forall i \in\{1, \ldots, I\}, \forall t \\
& 0 \leq \sum_{j=1}^{J} \mu_{i j}(t) \leq \mu_{\max }, \forall i \in\{1, \ldots, I\}, \forall j \in\{1, \ldots, J\}, \forall t \\
& 0 \leq X_{j}\left(\alpha_{j}(t)\right) \leq X_{j}^{\max }, \forall j \in\{1, \ldots, J\}, \forall t \\
& \bar{Q}<\infty
\end{aligned}
$$

However, traditional techniques, e.g., Markov Decision Theory and Dynamic Programming, require substantial statistics of system dynamics and suffer from high computational complexity [6]. By comparison, the recently developed Lyapunov optimization framework has shown its efficacy and efficiency in designing online control algorithms for such a constrained optimization of time-varying systems. It is a technique for solving problems of joint system stability and performance optimization on stochastic networks, especially communication and queueing systems. To achieve this goal, network algorithms are deigned to make control actions that greedily minimize a bound on the following drift-plus-penalty expression in each time slot $t$ :

$$
\Delta(t)+V F(t)
$$

where $\Delta(t)$ (Lyapunov drift) represents the congestion state of queue backlog, $F(t)$ denotes the objective function to be optimized, and $V$ is a non-negative weight that is chosen as desired to affect a performance tradeoff between backlog reduction and penalty minimization. Unlike the traditional techniques, Lyapunov optimization does not require knowledge of the statistics of relevant stochastic models to make online control decisions. The Lyapunov optimization algorithms commonly have a better computational complexity, and are easy to be implemented in practical systems [11][12][13]. In the following section, we will present an online control algorithm to solve problem in (4) based on the Lyapunov optimization framework. 


\section{Online Control Algorithm EESE}

Let $\mathbf{Q}(t) \triangleq(\mathbf{W}(t), \mathbf{M}(t))$ be a concatenated vector of all $W_{i}(t)$ and $M_{j}(t)$ queues. As a scalar measure of queue lengths, a quadratic Lyapunov function is defined as:

$$
L(\mathbf{Q}(t)) \triangleq \frac{1}{2} \sum_{i=1}^{I}\left[W_{i}(t)\right]^{2}+\frac{1}{2} \sum_{j=1}^{J}\left[M_{j}(t)\right]^{2}
$$

Then, the one-slot conditional Lyapunov drift $\Delta(\mathbf{Q}(t))$ is defined as:

$$
\Delta(\mathbf{Q}(t)) \triangleq \mathbb{E}[L(\mathbf{Q}(t+1))-L(\mathbf{Q}(t)) \mid \mathbf{Q}(t)]
$$

Following the drift-plus-penalty framework in Lyapunov optimization, EESE is designed to make decisions on $\mu_{i j}$ and $\alpha_{j}(t)$ to minimize upper bound on the following drift-pluspenalty term in each time slot:

$$
\Delta(\mathbf{Q}(t))+V \mathbb{E}\left\{\sum_{j=1}^{J} f_{j}(t) \mid \mathbf{Q}(t)\right\}
$$

A key derivation step is to obtain an upper bound on this term, which is defined as follows.

Theorem 1. (Drift-plus-Penalty Bound) Under any control algorithm, the drift-plus-penalty expression has the following upper bound for all $t$, all possible values of $\mathbf{Q}(t)$, and all parameters $V \geq 0$ :

$$
\begin{array}{r}
\Delta(\mathbf{Q}(t))+V \mathbb{E}\left\{\sum_{j=1}^{J} f_{j}(t) \mid \mathbf{Q}(t)\right\} \leq B+\mathbb{E}\left\{\sum_{i=1}^{I} W_{i}(t) A_{i}(t) \mid \mathbf{Q}(t)\right\} \\
+\mathbb{E}\left\{\sum_{i=1}^{I} \sum_{j=1}^{J} \mu_{i j}(t)\left[M_{j}(t)-W_{i}(t)\right] \mid \mathbf{Q}(t)\right\} \\
+\mathbb{E}\left\{\sum_{j=1}^{J}\left[V f_{j}(t)-M_{j}(t) X_{j}(t)\right] \mid \mathbf{Q}(t)\right\}
\end{array}
$$

where $B=\frac{I\left(A_{\max }^{2}+\mu_{\max }^{2}\right)+J\left(X_{\max }^{2}+I \mu_{\max }^{2}\right)}{2}$.

\section{Proof:}

Squaring both sides of the queueing dynamic (1), and using the fact that for any $Q \geq 0, b \geq 0, A \geq 0,(\max [Q-b, 0]+$ $A)^{2} \leq Q^{2}+A^{2}+b^{2}+2 Q(A-b)$, we have:

$$
\begin{array}{r}
{\left[W_{i}(t+1)\right]^{2} \leq\left[W_{i}(t)\right]^{2}+\left[A_{i}(t)\right]^{2}+\left[\sum_{j=1}^{J} \mu_{i j}(t)\right]^{2}} \\
+2 W_{i}(t)\left[A_{i}(t)-\sum_{j=1}^{J} \mu_{i j}(t)\right]
\end{array}
$$

Summing the above over $i=1, \ldots, I$ and using the fact that, we have:

$$
\begin{array}{r}
\sum_{i=1}^{I}\left\{\left[W_{i}(t+1)\right]^{2}-\left[W_{i}(t)\right]^{2}\right\} \leq I\left(A_{\max }^{2}+\mu_{\text {max }}^{2}\right) \\
+2 \sum_{i=1}^{I} W_{i}(t)\left[A_{i}(t)-\sum_{j=1}^{J} \mu_{i j}(t)\right]
\end{array}
$$

Repeating the above steps for the queue $M_{j}(t)$, and by using the fact that $0 \leq X_{j}(t) \leq X_{\max }$, we have:

$$
\begin{array}{r}
\sum_{j=1}^{J}\left\{\left[M_{j}(t+1)\right]^{2}-\left[M_{j}(t)\right]^{2}\right\} \leq J\left(X_{\max }^{2}+I \mu_{\max }^{2}\right) \\
+2 \sum_{j=1}^{J} M_{j}(t)\left[\sum_{i=1}^{I} \mu_{i j}(t)-X_{j}(t)\right]
\end{array}
$$

Combining these two bounds together, and taking the expectation with respect to $\mathbf{Q}(t)$ on both sides, we arrive at the following one-slot conditional Lyapunov drift $\Delta(\mathbf{Q}(t))$ :

$$
\begin{array}{r}
\Delta(\mathbf{Q}(t)) \leq B+\mathbb{E}\left\{\sum_{i=1}^{I} W_{i}(t) A_{i}(t) \mid \mathbf{Q}(t)\right\} \\
+\mathbb{E}\left\{\sum_{i=1}^{I} \sum_{j=1}^{J} \mu_{i j}(t)\left[M_{j}(t)-W_{i}(t)\right] \mid \mathbf{Q}(t)\right\} \\
-\mathbb{E}\left\{\sum_{j=1}^{J} M_{j}(t) X_{j}(t) \mid \mathbf{Q}(t)\right\}
\end{array}
$$

where $B=\frac{I\left(A_{\max }^{2}+\mu_{\max }^{2}\right)+J\left(X_{\max }^{2}+I \mu_{\max }^{2}\right)}{2}$.

Now adding to both sides the penalty expression, i.e., the term $V \mathbb{E}\left\{\sum_{j=1}^{J} f_{j}(t) \mid \mathbf{Q}(t)\right\}$, proves the theorem.

Minimizing the right-hand-side of (8) is equivalen$\mathrm{t}$ to minimizing $\mathbb{E}\left\{\sum_{i=1}^{I} \sum_{j=1}^{J} \mu_{i j}(t)\left[M_{j}(t)-W_{i}(t)\right] \mid \mathbf{Q}(t)\right\}+$ $\mathbb{E}\left\{\sum_{j=1}^{J}\left[V f_{j}(t)-M_{j}(t) X_{j}(t)\right] \mid \mathbf{Q}(t)\right\}$ under the same constraints in (4). Therefore, we can design the control algorithm as in Algorithm 1.

The following theorem presents bounds on the time average energy cost and queue backlogs achieved by our EESE algorithm.

Theorem 2. (Algorithm Performance) Define $\boldsymbol{\Lambda}$ as the set of all rate vectors that satisfy the constraints in (4). For any rate vector $\lambda \in \Lambda$, suppose there exists an $\epsilon>0$ such that $\lambda+\epsilon \mathbf{1} \in \mathbf{\Lambda}$, then under our algorithm we have:

$\bar{Q} \triangleq \lim _{T \rightarrow \infty} \frac{1}{T} \sum_{t=0}^{T-1}\left[\mathbb{E}\left\{\sum_{i=1}^{I} W_{i}(t)\right\}+\mathbb{E}\left\{\sum_{j=1}^{J} M_{j}(t)\right\}\right] \leq \frac{B+V \bar{F}_{\max }}{\epsilon}$ 


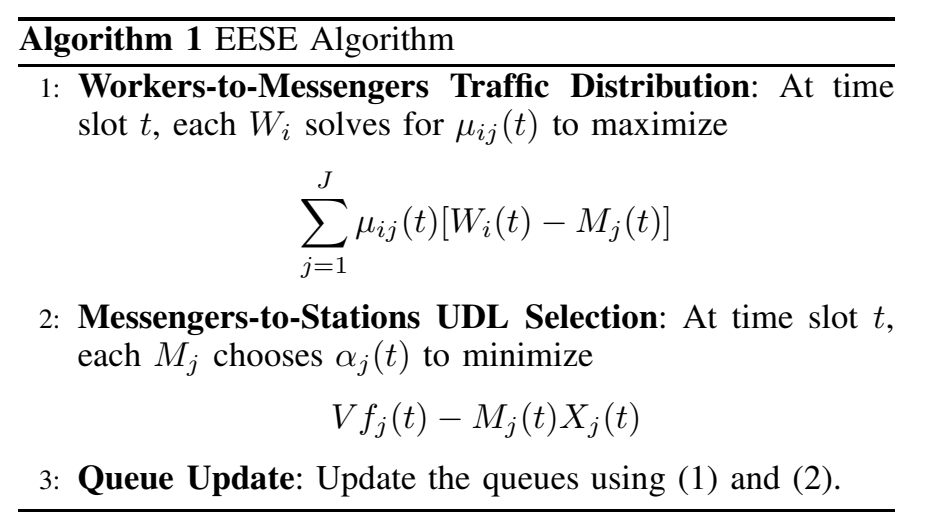

$$
\bar{F} \triangleq \lim _{T \rightarrow \infty} \frac{1}{T} \sum_{t=0}^{T-1} \mathbb{E}\left\{\sum_{j=1}^{J} f_{j}(t)\right\} \leq \bar{F}^{*}+\frac{B}{V}
$$

Here $\bar{F}_{\max }$ and $\bar{F}^{*}$ are the maximal value and the optimal value for the cost defined in (4) respectively, and 1 denotes the vector of all 1's.

Proof: According to Caratheodory's theorem [6][11], we can easily prove that there exists a randomized stationary control policy $\pi$ that chooses feasible control decisions $\mu_{i j}$ and $\alpha_{j}(t)$, independent of the current queue backlogs, and achieves the following guarantees:

$$
\begin{gathered}
\mathbb{E}\left\{\sum_{j=1}^{J} f_{j}^{\pi}(t)\right\}=\bar{F}^{*} \\
\mathbb{E}\left\{A_{i}^{\pi}(t)\right\}=\mathbb{E}\left\{\sum_{j=1}^{J} \mu_{i j}^{\pi}(t)\right\} \\
\mathbb{E}\left\{\sum_{i=1}^{I} \mu_{i j}^{\pi}(t)\right\}=\mathbb{E}\left\{X_{j}^{\pi}(t)\right\}
\end{gathered}
$$

Because, every time slot $t$, our implementation seeks to minimize the right-hand-side of the drift-plus-penalty expression in (8):

$$
\begin{array}{r}
\Delta(\mathbf{Q}(t))+V \mathbb{E}\left\{\sum_{j=1}^{J} f_{j}(t) \mid \mathbf{Q}(t)\right\} \leq B+V \mathbb{E}\left\{\sum_{j=1}^{J} f_{j}^{\dagger}(t) \mid \mathbf{Q}(t)\right\} \\
+\mathbb{E}\left\{\sum_{i=1}^{I} W_{i}(t)\left[A_{i}(t)-\sum_{j=1}^{J} \mu_{i j}^{\dagger}(t)\right] \mid \mathbf{Q}(t)\right\} \\
+\mathbb{E}\left\{\sum_{j=1}^{J} M_{j}(t)\left[\sum_{i=1}^{I} \mu_{i j}(t)-X_{j}\left(\alpha_{j}^{\dagger}(t)\right)\right] \mid \mathbf{Q}(t)\right\}
\end{array}
$$

where $f_{j}^{\dagger}(t), \mu_{i j}^{\dagger}(t)$ and $\alpha_{j}^{\dagger}(t)$ are the resulting decisions and attributed values under any alternative (possibly randomized) policy (denoted by $\dagger$ ). Now since $\lambda+\epsilon \mathbf{1} \in \boldsymbol{\Lambda}$, it can be known from (11)-(13) that there exists a stationary and randomized policy $\pi^{\prime}$ that achieves the following:

$$
\begin{gathered}
\mathbb{E}\left\{\sum_{j=1}^{J} f_{j}^{\pi^{\prime}}(t)\right\}=\bar{F}^{*}(\lambda+\epsilon \mathbf{1}) \\
\mathbb{E}\left\{A_{i}^{\pi^{\prime}}(t)\right\}=\mathbb{E}\left\{\sum_{j=1}^{J} \mu_{i j}^{\pi^{\prime}}(t)\right\}-\epsilon \\
\mathbb{E}\left\{\sum_{i=1}^{I} \mu_{i j}^{\pi^{\prime}}(t)\right\}=\mathbb{E}\left\{X_{j}^{\pi^{\prime}}(t)\right\}-\epsilon
\end{gathered}
$$

Here $\bar{F}^{*}(\lambda+\epsilon \mathbf{1})$ is the minimum cost corresponding to the rate vector $\lambda+\epsilon \mathbf{1}$. Plugging (15)-(17) into the right-hand-side of (14) yields:

$$
\begin{array}{r}
\Delta(\mathbf{Q}(t))+V \mathbb{E}\left\{\sum_{j=1}^{J} f_{j}(t) \mid \mathbf{Q}(t)\right\} \leq B+V \bar{F}^{*}(\lambda+\epsilon \mathbf{1}) \\
-\epsilon \sum_{i=1}^{I} W_{i}(t)-\epsilon \sum_{j=1}^{J} M_{j}(t)
\end{array}
$$

Now we can take expectations on both sides over $\mathbf{Q}(t)$ to get:

$$
\begin{array}{r}
\mathbb{E}\{L(\mathbf{Q}(t+1))-L(\mathbf{Q}(t))\}+V \mathbb{E}\left\{\sum_{j=1}^{J} f_{j}(t) \mid \mathbf{Q}(t)\right\} \leq B \\
\quad+V \bar{F}^{*}(\lambda+\epsilon \mathbf{1})-\epsilon \mathbb{E}\left\{\sum_{i=1}^{I} W_{i}(t)\right\}-\epsilon \mathbb{E}\left\{\sum_{j=1}^{J} M_{j}(t)\right\}
\end{array}
$$

Rearranging the terms, and using the fact that $0 \leq \bar{F}^{*}(\lambda+$ $\epsilon \mathbf{1}) \leq \bar{F}_{\max }$ we get that:

$$
\begin{array}{r}
\mathbb{E}\{L(\mathbf{Q}(t+1))-L(\mathbf{Q}(t))\}+\epsilon\left(\mathbb{E}\left\{\sum_{i=1}^{I} W_{i}(t)\right\}\right. \\
\left.+\mathbb{E}\left\{\sum_{j=1}^{J} M_{j}(t)\right\}\right) \leq B+V \bar{F}_{\max }
\end{array}
$$

Summing the above over $t=0,1, \ldots, T-1$, rearranging the terms, using the fact that $L(\mathbf{Q}(t)) \geq 0$ for all $t$, and dividing both sides by $\epsilon T$, we have:

$$
\frac{1}{T} \sum_{t=0}^{T-1}\left[\mathbb{E}\left\{\sum_{i=1}^{I} W_{i}(t)\right\}+\mathbb{E}\left\{\sum_{j=1}^{J} M_{j}(t)\right\}\right] \leq \frac{B+V \bar{F}_{\max }}{\epsilon}
$$

which proves (9) by taking limit as $T \rightarrow \infty$.

To prove (10), using (18), we have:

$$
V \mathbb{E}\left\{\sum_{j=1}^{J} f_{j}(t) \mid \mathbf{Q}(t)\right\} \leq V \bar{F}^{*}(\lambda+\epsilon \mathbf{1})+B
$$


Summing the above over $t=0, \ldots, T-1$, and dividing both sides by $T V$, we have:

$$
\frac{1}{T} \sum_{t=0}^{T-1} \mathbb{E}\left\{\sum_{j=1}^{J} f_{j}(t)\right\} \leq \bar{F}^{*}(\lambda+\epsilon \mathbf{1})+\frac{B}{V}
$$

which proves (10) by taking limit as $T \rightarrow \infty$ and letting $\epsilon \rightarrow 0$.

The following theorem shows that EESE is robust when it makes control decisions based on the queue backlog estimates $\hat{\mathbf{Q}}(t)$ that differ from the actual queue backlogs.

Theorem 3. (Algorithm Robustness) Suppose there exists an $\epsilon>0$ such that $\lambda+\epsilon \mathbf{1} \in \Lambda$. Also suppose there exists a constant $c_{e}$, such that at all time $t$, the estimated backlog sizes $\hat{W}_{i}(t), \hat{M}_{j}(t)$ and the actual backlog sizes $W_{i}(t), M_{j}(t)$ satisfy $\left|\hat{W}_{i}(t)-W_{i}(t)\right| \leq c_{e}$ and $\left|\hat{M}_{j}(t)-M_{j}(t)\right| \leq c_{e}$. Then under our algorithm, we have:

$\bar{Q} \triangleq \lim _{T \rightarrow \infty} \frac{1}{T} \sum_{t=0}^{T-1}\left[\mathbb{E}\left\{\sum_{i=1}^{I} W_{i}(t)\right\}+\mathbb{E}\left\{\sum_{j=1}^{J} M_{j}(t)\right\}\right] \leq \frac{B^{\prime}+V \bar{F}_{\max }}{\epsilon}$

$$
\bar{F} \triangleq \lim _{T \rightarrow \infty} \frac{1}{T} \sum_{t=0}^{T-1} \mathbb{E}\left\{\sum_{j=1}^{J} f_{j}(t)\right\} \leq \bar{F}^{*}+\frac{B^{\prime}}{V}
$$

Here $B^{\prime}=B+c_{e}\left(I A_{\max }+I \mu_{\max }+J I \mu_{\max }+J X_{\max }\right)$.

Proof: It suffices to show that using $\hat{W}_{i}(t), \hat{M}_{j}(t)$, we still minimize the right-hand-side of the drift expression in (8) to within some additive constant. Denote $e_{i}^{W}(t)=\hat{W}_{i}(t)-W_{i}(t)$ and $e_{j}^{M}(t)=\hat{M}_{j}(t)-M_{j}(t)$. Suppose now $\hat{W}_{i}(t)$ and $\hat{M}_{j}(t)$ are used to carry out the algorithm, then we see that we try to minimize:

$$
\begin{array}{r}
\bar{F}(\hat{\mathbf{Q}}(t)) \triangleq V \mathbb{E}\left\{\sum_{j=1}^{J} f_{j}(t) \mid \mathbf{Q}(t)\right\} \\
+\mathbb{E}\left\{\sum_{i=1}^{I} W_{i}(t)\left[A_{i}(t)-\sum_{j=1}^{J} \mu_{i j}(t)\right] \mid \mathbf{Q}(t)\right\} \\
+\mathbb{E}\left\{\sum_{j=1}^{J} M_{j}(t)\left[\sum_{i=1}^{I} \mu_{i j}(t)-X_{j}(t)\right] \mid \mathbf{Q}(t)\right\} \\
+\mathbb{E}\left\{\sum_{i=1}^{I} e_{i}^{W}(t)\left[A_{i}(t)-\sum_{j=1}^{J} \mu_{i j}(t)\right] \mid \mathbf{Q}(t)\right\} \\
+\mathbb{E}\left\{\sum_{j=1}^{J} e_{j}^{M}(t)\left[\sum_{i=1}^{I} \mu_{i j}(t)-X_{j}(t)\right] \mid \mathbf{Q}(t)\right\}
\end{array}
$$

Using the fact that $\left|e_{i}^{W}(t)\right| \leq c_{e}$ and $\left|e_{j}^{M}(t)\right| \leq c_{e}$, and the fact that $\left|A_{i}(t)-\sum_{j=1}^{J} \mu_{i j}(t)\right| \leq A_{\max }+\mu_{\max }, \mid \sum_{i=1}^{I} \mu_{i j}(t)-$ $X_{j}(t) \mid \leq I \mu_{\max }+X_{\max }$, we know that

$$
\bar{F}(\hat{\mathbf{Q}}(t)) \leq \bar{F}(\mathbf{Q}(t))+c_{e}\left(I A_{\max }+I \mu_{\max }+J I \mu_{\max }+J X_{\max }\right)
$$

This shows that (8) holds with $\mathbf{Q}(t)$ replaced by $\hat{\mathbf{Q}}(t)$, and $B$ replaced by $B^{\prime}=B+c_{e}\left(I A_{\max }+I \mu_{\max }+J I \mu_{\max }+\right.$ $\left.J X_{\max }\right)$. The rest of the proof follows similarly as the proof of Theorem 2.

\section{CONCLUSIONS}

This paper develops a control algorithm EESE for reducing energy consumption on data communication for satellite swarm systems. EESE can independently and simultaneously make decisions on traffic dispatching control actions on both ISLs and UDLs. In particular, EESE can approach the optimal cost within provable $\mathcal{O}(1 / V)$ deviation with a worst-case delay tradeoff that is $\mathcal{O}(V)$, without requiring substantial statistics of system dynamics. Besides, we prove that EESE is robust against estimation errors in traffic load. Furthermore, EESE is computationally efficient and easy to implement in large swarm-based satellite systems.

This work is our primary study on such a research issue. In the future, we hope to implement EESE in our STK-based simulation environment, as well as in our experimental swarm prototype, to test and evaluate its performance.

\section{REFERENCES}

[1] S. Nag and L. Summerer, "Behaviour based, autonomous and distributed scatter manoeuvres for satellite swarms," Acta Astronautica, vol. 82, no. 1, pp. 95 - 109, 2013.

[2] E. Vassev, R. Sterritt, C. Rouff, and M. Hinchey, "Swarm technology at nasa: Building resilient systems," IT Professional, vol. 14, no. 2, pp. 36-42, 2012.

[3] P. D'Arrigo and S. Santandrea, "The apies mission to explore the asteroid belt," Advances in Space Research, vol. 38, no. 9, pp. 2060 - 2067, 2006. [Online]. Available: http://www.sciencedirect.com/science/article/pii/S0273117706005084

[4] J. J. Puschell and E. Stanton, "Cubesat modules for multispectral environmental imaging from polar orbit," in Proc. SPIE 8516, Remote Sensing System Engineering IV, vol. 8516, 2012, pp. $85160 \mathrm{~B}: 1-$ 85 160B:8.

[5] C. Verhoeven, M. Bentum, G. Monna, J. Rotteveel, and J. Guo, "On the origin of satellite swarms," Acta Astronautica, vol. 68, no. 7-8, pp. 1392 - 1395, 2011.

[6] M. J. Neely, Stochasitic Network Optimization with Application to Communication and Queueing Systems, J. Walrand, Ed. Morgan \& Claypool, 2010.

[7] T. Vladimirova, X. Wu, and C. Bridges, "Development of a satellite sensor network for future space missions," in IEEE Aerospace Conference 2008, 2008, pp. 1-10.

[8] P. Muri and J. McNair, "A survey of communication sub-systems for intersatellite linked systems and cubesat missions," Journal of Communications, vol. 7, no. 4, pp. 290-308, 2012.

[9] S. S. Anderson, L. H. Hogle, R. Breitwisch, C. Edwards, R. J. Hamilton, and D. W. Lipke, "Aeronautical satellite data link concept, design, and flight test results," Acta Astronautica, vol. 14, no. 0, pp. 323 - 338, 1986.

[10] J. Zhu and S. Roy, "Improving link layer performance on satellite channels with shadowing via delayed two-copy selective repeat arq," IEEE Journal on Selected Areas in Communications, vol. 22, no. 3, pp. 472-481, 2004.

[11] Y. Yao, L. Huang, A. Sharma, L. Golubchik, and M. Neely, "Data centers power reduction: A two time scale approach for delay tolerant workloads," in Proceedings IEEE INFOCOM 2012, Orlando, FL, USA, 2012, pp. 1431-1439. 
[12] R. Urgaonkar, B. Urgaonkar, M. J. Neely, and A. Sivasubramaniam, "Optimal power cost management using stored energy in data centers," in Proceedings of the ACM SIGMETRICS joint international conference on Measurement and modeling of computer systems, New York, NY, USA, 2011, pp. 221-232.

[13] M. Neely, "Opportunistic scheduling with worst case delay guarantees in single and multi-hop networks," in Proceedings IEEE INFOCOM 2011, Shanghai, China, 2011, pp. 1728-1736.

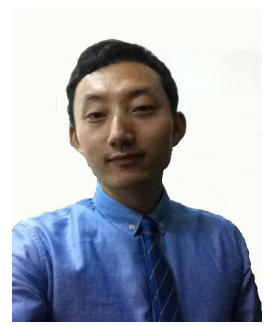

Yuan An was born at Shandong, China, in 1982. $\mathrm{He}$ got his $\mathrm{PhD}$ degree on Computer Science from University of Chinese Academy of Sciences, China, in 2010 . He is now a researcher at State key Lab of Astronautic Dynamics, Xi'an Satellite Control Center, China. His research interests include satellite communication, wireless network, and satellite swarming technologies.

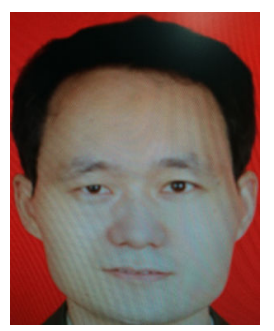

Jiancheng Li was born at Wuhan, China, in 1971. $\mathrm{He}$ got his $\mathrm{PhD}$ degree on Computer Science from Northwestern Polytechnical University, China, in 1993. He is now an advanced researcher at State key Lab of Astronautic Dynamics, Xi'an Satellite Control Center, China. His research interests include satellite communication, wireless network, and satellite swarming technologies.

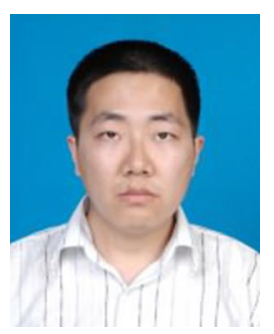

Weiwei Fang was born at Wuhu, China, in 1981. $\mathrm{He}$ got his PhD degree on Computer Science from Beihang University, China, in 2010 . He is now an associate professor at Beijing Jiaotong University. His research interests include satellite communication, wireless network, and satellite swarming technologies.

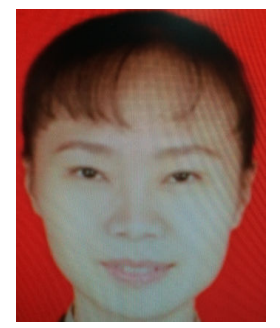

Baohua Wang was born at Beijing, China, in 1972 $\mathrm{He}$ got his Master degree on Computer Science from Xi' an Jiaotong University, China, in 1985. He is now an advanced researcher at State key Lab of Astronautic Dynamics, Xi'an Satellite Control Center, China. His research interests include satellite communication, wireless network, and satellite swarming technologies.

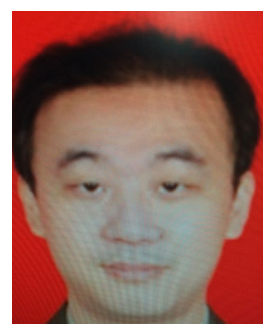

Qiwang Guo was born at Tianjin, China, in 1972. He got his Master degree on Computer Science from Shanghai Jiaotong University, China, in 2007. He is now a researcher at State key Lab of Astronautic Dynamics, Xi' an Satellite Control Center, China. His research interests include satellite communication, wireless network, and satellite swarming technologies.

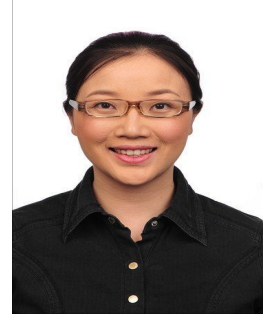

Jing Li was born at Datong, China, in 1969. She got his $\mathrm{PhD}$ degree on Computer Science from National University of Defence Technology, China, in 2005. She is now a researcher at State key Lab of Astronautic Dynamics, Xi' an Satellite Control Center, China. Her research interests include satellite communication, wireless network, and satellite swarming technologies.

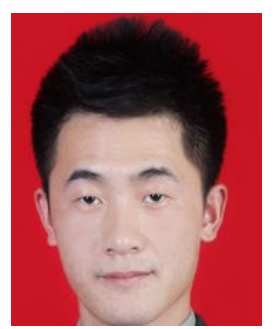

Xiaolong Li was born at Mudanjiang, China, in 1987. He got his Bachelor degree on Electronic Engineering from Xi'an Jiaotong University, China, in 2012. He is now a researcher at State key Lab of Astronautic Dynamics, Xi'an Satellite Control Center, China. His research interests include satellite communication, wireless network, and satellite swarming technologies.

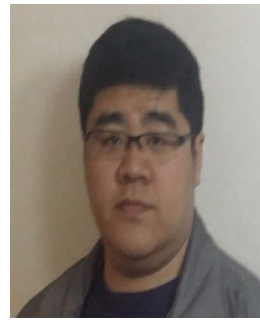

Xing Du was born at Shanghai, China, in 1982. He got his $\mathrm{PhD}$ degree on Electronic Engineering from Sichuan University, China, in 2012. He is now a researcher at State key Lab of Astronautic Dynamics, Xi' an Satellite Control Center, China. His research interests include satellite communication, wireless network, and satellite swarming technologies. 